\title{
REPRODUÇÃO E POTENCIAIS IMPACTOS SOBRE LITTORINA FLAVA (NEOGASTROPODA: LITTORINIDAE) NA ILHA DO MEL, PARANÁ
}

\author{
FERREIRA-JR, A.L. ${ }^{1 *} ;$ CARVALHO, I.F.P. ${ }^{2} ;$ ABSHER, T.M. ${ }^{2} \&$ CRISTO, S. W. ${ }^{3}$ \\ 1 - Universidade Federal de Santa Catarina \\ 2 - Laboratório de Moluscos Marinhos CEM/UFPR. Av. Beira mar s/n 83255-000 Pontal do Sul, Paraná \\ 3 - Departamento de Biologia Geral, Universidade Estadual de Ponta Grossa, Ponta Grossa, Paraná \\ *Corresponding author: alfjr_1@hotmail.com
}

\begin{abstract}
Ferreira-Jr, A.L.; Carvalho, I.F.P.; Absher, T.M. \& Susete, C. W. 2014. Reproduction and potential impacts on Littorina flava (Neogastropoda: Littorinidae) at Mel Island, Paraná. Braz. J. Aquat. Sci. Technol. 18(2): 53-57. elSSN 19839057. DOI: 10.14210/bjast.v18n2.p53-57 Littorina flava is used as bioindicator to measure environmental impacts and to assess the quality of the environment and how it changes over time. The main objective of this work was to determine the reproductive characteristics of $L$. flava and its relationship with environmental variables and the influence of contaminants in a rocky coast of Mel Island, Paraná, Brazil. Monthly sampling during eight months were performed in the intertidal region during spring low tide in two periods (Winter/Spring and Summer/Fall) in three levels (Upper, Middle and Lower) with three replicates. Monthly 40 individuals of $L$. flava were collected in the three levels. Environmental parameters (air and water temperature and water salinity) were also obtained. The organisms' height and width were measured, examined for sex identification and gonadal maturation stage. Gonad maturation of $L$. flava was verified in the end of the first sampling cycle (Winter/Spring). Sexual intercourse behavior was observed in September and October 2010. This reproductive behavior was less observed in the second period (Summer/Fall) together with a decrease in matured gonads. Sexual proportion demonstrated high predominance of males, indicating a possible imposex occurrence due to pollutants verified in the area (fuel from boats, sewer discharge).
\end{abstract}

Key words: reproduction, molluscs, Paranaguá Estuarine Complex, imposex, organotin.

\section{INTRODUÇÃO}

Os gastrópodes são um dos grupos mais abundantes em regiões entremarés de substratos rochosos. Os principais representantes de gastrópodes nesses costões pertencem à família Littorinidae (Borkowski, 1971). Dentre as espécies que constituem esta família, destacam-se na costa sul brasileira: Littorina flava King \& Broderip, 1832, Littorina lineata Orbigny, 1841, Littorina lineolata Orbigny, 1840, Littorina ziczac (Gmelin, 1791), e Littorina angulifera (Lamarck, 1822) (Rios, 2009). Esses gastrópodes podem ser utilizados como indicadores da região do intermareal em costões rochosos (Bauer et al., 1995). Além disso, as espécies do gênero Littorina podem ser utilizadas para monitorar a contaminação ambiental (Broeck et al.; 2009). É certo que metais pesados e/ou compostos orgânicos podem afetar características reprodutivas e fisiológicas de gastrópodes (Castro et al., 2000; 2007a; 2007b; 2012; Broeck et al., 2009; Biasi et al., 2010).

Littorina flava é herbívora, com ocorrência desde o Maranhão a Torres - RS e é considerada como uma das maiores espécies da família $(18 \times 12 \mathrm{~mm})$ (Rios, 2009). Esta espécie é dióica com fecundação interna e produção de cápsulas pelágicas com ovos envoltos em massas gelatinosas (Hyman, 1967; Borkowski, 1971; Simone, 1998). Em decorrência da sua ampla distribuição no litoral brasileiro e ocorrência em locais intensamente afetados por atividades antrópicas, $L$. flava pode ser utilizada como organismo indicador de degradação ambiental. No entanto, para que se possa utilizá-la como bioindicadora ambiental, faz-se necessário estudar as características reprodutivas da espécie próximo a regiões com riscos ambientais. Um destes locais com potencial a desastres ambientais são regiões portuárias.

O litoral do Paraná possui dois portos: Paranaguá e Antonina, responsáveis por escoamento de diversos produtos para outras regiões brasileiras e mundiais (Noernberg et al., 2008). Além dos portos, próximo ao canal de acesso do setor portuário localiza-se a Ilha do Mel. Esta possui dois locais de embarque e desembarque de passageiros e mercadorias, realizados por pequenas embarcações (responsáveis por resíduos de combustível). Além disto, a llha do Mel possui um Centro de Resíduo Sólido (CRS) próximo a um destes embarcadouros e ambos (embarcações e CRS) representam riscos ambientais para a fauna local (Schena, 2006; Castro et al., 2012). Castro et al., (2007) constataram a ocorrência de imposex em Thais haemastoma (Linnaeus, 1767) na Ilha do Mel em locais próximos aos embarcadouros. Estudos efetuados com Littorina littorea (Linnaeus, 1758) em regiões portuárias da Bélgica, constataram a presença de organismos com imposex e castração de fêmeas (Broeck et al., 2009). Além disto, no litoral brasileiro são escassos os estudos de ciclo reprodutivos de gastrópodes (Matthews-Cascon \& Martins, 1999; Rocha-Barreira, 2002; Rocha-Barreira, 2010). 
Portanto, este trabalho tem como objetivo analisar características reprodutivas de $L$. flava em um costão rochoso da llha do Mel (Paraná) relacionando-as com a variação sazonal e influência de contaminantes.

\section{MATERIAIS E MÉTODOS}

\section{Área de Estudo}

O litoral do Paraná, localizado no sul do Brasil, estende-se desde a Vila de Ararapira ao norte (2512'44"S; 4801'15"W) até a barra do Rio SaíGuaçu, ao sul (2558'38'S; 48³5'26”W). Este litoral possui $146,6 \mathrm{~km}$ de costões rochosos e enrocamentos abrigados, representando $8,75 \%$ da costa paranaense (Noernberg et al., 2008). Apresenta dois estuários, o Complexo Estuarino de Paranaguá (CEP) e a Baía de Guaratuba, sendo o primeiro o maior (Figura 1).

O CEP sofre, a leste, influência do oceano Atlântico através das barras Norte e Sueste e é circundado por bacias hidrográficas com cerca de $3361 \mathrm{~km}^{2}$ (Maack, 1981). Este sistema estuarino possui dois portos, Paranaguá e Antonina, que escoam cargas líquidas (óleos), granel sólido, fertilizantes e cargas em geral (Noernberg et al., 2008). Além dos portos, o CEP possui como outro centro econômico uma das referenciais turísticas do Estado do Paraná - a llha do Mel, localizada na desembocadura do complexo. Os locais de embarque e desembarque da ilha são os trapiches das vilas da Nova Brasília e das Encantadas, esta última

Figura 1 - Localização dos pontos de coleta de L. flava em um costão rochoso entremarés (o) na Praia de Encantadas - Ilha do Mel, Complexo Estuarino de Paranaguá, PR.

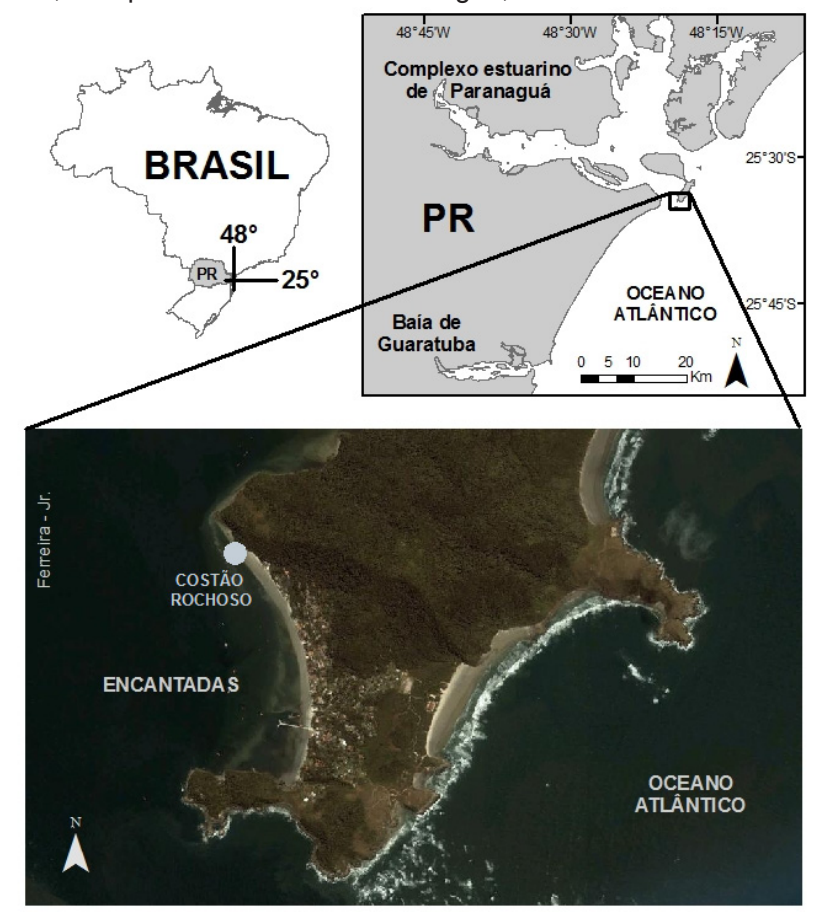

vila possui o maior número de moradores, somando aproximadamente 800 residentes (Schena, 2006). $\mathrm{Na}$ extremidade da praia existente na frente da Vila das Encantadas, situa-se o Centro de Resíduo Sólido da Ilha do Mel, sendo que o presente estudo foi feito no costão rochoso situado ao lado deste (Figura 1).

\section{Amostragem}

Foram realizadas oito coletas durante dois períodos: julho a outubro de 2010 (Inverno/Primavera) e janeiro a abril de 2011 (Verão/Outono) em três níveis da região entremarés (Superior, Médio e Inferior) com três transectos ( $A, B$ e $C$ ) sendo que cada um destes seria uma réplica para cada nível. As amostragens foram efetuadas na baixamar de maré de sizígia. Mensalmente foram coletados 40 indivíduos de $L$. flava distribuídos nos três níveis amostrais. Os parâmetros ambientais monitorados in situ foram temperatura do ar, da água e salinidade.

\section{Metodologia laboratorial}

Em laboratório foi primeiramente efetuada a biometria dos exemplares: altura (A) e largura (L), com o uso de um paquímetro com precisão de $0,5 \mathrm{~mm}$. Posteriormente, foi retirada a concha de cada exemplar e efetuada a análise visual das gônadas. Estas foram classificadas em quatro estágios de repleção, de acordo com o grau de cobertura (sobreposição) do tecido gonádico sobre a glândula digestiva (GD), sendo: (V) vazia: 0-25\%; (PV) parcialmente vazia: 25-50\%; (PC) parcialmente cheia: 50-75\%; (C) cheia: 75-100\%; (NI) não identificado (adaptado de RochaBarreira, 2002). A sexagem foi feita por análise a fresco, mediante raspagem das gônadas e observação das células sexuais em microscópio óptico.

\section{RESULTADOS}

A salinidade apresentou variação de 22 a 32 , a temperatura do ar de 16,5 a $26,0^{\circ} \mathrm{C}$ e a temperatura da água 17,5 a $26,5^{\circ} \mathrm{C}$. Valores mais baixos da temperatura do ar e da água foram observados no primeiro período (Inverno/Primavera) $\left(16,5\right.$ a $\left.21,5^{\circ} \mathrm{C}\right)$ e mais elevados no segundo período (Verão/Outono) (22 a $\left.26,5^{\circ} \mathrm{C}\right)$.

Durante este estudo, 320 exemplares de $L$. flava foram analisados. A altura média foi de 11,32 ( \pm 3,92 ) $\mathrm{mm}$, com variação de 2 a $19 \mathrm{~mm}$ e a largura média foi de 8,61 ( $\pm 2,96$ ) $\mathrm{mm}$, com variação de 1 a $14 \mathrm{~mm}$.

A caracterização sexual demonstrou alta predominância de machos (280 indivíduos $=87,5 \%$ ) em relação às fêmeas (18 indivíduos $=5,62 \%$ ) e não identificados (22 indivíduos $=6,88 \%$ ), resultando em 
Tabela 1 - Número de indivíduos por sexo e proporção sexual (PS) de L. flava nos diversos meses de amostragem. M - machos; F fêmeas; $\mathrm{NI}$ - não identificados.

\begin{tabular}{|c|c|c|c|c|c|c|c|c|c|}
\hline \multirow{2}{*}{$\begin{array}{l}\text { Período } \\
\text { Meses }\end{array}$} & \multicolumn{4}{|c|}{$1^{\circ}$} & \multicolumn{4}{|c|}{$2^{\circ}$} & \multirow{2}{*}{ Total } \\
\hline & $\overline{\mathrm{jul} / 10}$ & ago/10 & set/10 & out/10 & jan/11 & fev/11 & $\mathrm{mar} / 11$ & $a b r / 11$ & \\
\hline $\mathbf{M}$ & 36 & 34 & 36 & 30 & 35 & 33 & 37 & 39 & 280 \\
\hline$F$ & 2 & 1 & 2 & 6 & 2 & 5 & 0 & 1 & 18 \\
\hline NI & 2 & 5 & 3 & 4 & 3 & 2 & 3 & 0 & 22 \\
\hline PS & 18:1 & $34: 1$ & 18:1 & $5: 1$ & $17,5: 1$ & $6,6: 1$ & $37: 0$ & $39: 1$ & $15,5: 1$ \\
\hline
\end{tabular}

proporção sexual macho: fêmea (M:F) de 15,5: 1 (Tabela 1).

Em relação à repleção gonádica, $32,5 \%$ dos exemplares apresentaram gônadas cheias, $26,56 \%$ parcialmente cheias, $21,25 \%$ parcialmente vazias e $12,81 \%$ vazias, sendo que em $12,81 \%$ não foi possível à identificação. No primeiro período (Inverno/ Primavera) foram verificadas porcentagens mais altas de gônadas repletas e nos meses de setembro e outubro também foi observado em campo o comportamento de cópula. Este comportamento reprodutivo foi menos observado no segundo período de estudo (Verão/Outono) juntamente com a diminuição das gônadas (Figura 2).

\section{DISCUSSÃO}

A repleção gonádica durante os períodos estudados demonstraram gônadas mais repletas entre julho e outubro (Inverno/Primavera) em relação ao período de janeiro a abril (Verão/Outono). Borkowski (1971), em um estudo realizado na Flórida (EUA), verificou período de desova de cinco espécies da família Littorinidae: Littorina lineata, Littorina lineolata, Nodilittorina tuberculata (Menke, 1828), Tectarius muricatus (Linnaeus, 1758) e L. ziczac, durante os meses com temperaturas da água mais elevadas ( 25 a $30^{\circ} \mathrm{C}$ ). Esta característica de espécies da referida família desovarem durante períodos de temperaturas mais altas (de 23 a $26,5^{\circ} \mathrm{C}$ ) foi encontrada no presente estudo. Uma menor repleção das gônadas de L. flava foi observada durante o Verão/Outono e durante a primavera houve predomínio de gônadas repletas. Possivelmente a elevação da temperatura ocasionou a desova e em consequência gônadas mais vazias de L. flava no período.

Segundo Rocha-Barreira (2002), Lottia subrugosa (Orbigny, 1846) em uma região de baixa latitude do Brasil (Ceará), apresentou períodos de maturação (gônadas cheias) de fevereiro a junho e de agosto a fevereiro, com desovas em julho e em março. Esta mesma autora associou o período de desova à diminuição da salinidade que na latitude do presente estudo ocorre no verão devido à maior precipitação atmosférica. Para Neritina virginea (Linnaeus,

Figura 2 - Frequência relativa mensal dos diversos estágios gonádicos de L. flava coletadas na llha do Mel, Paraná, nos dois períodos de amostragem (1 - julho a outubro de 2010 e 2 - janeiro a abril de 2011). C - cheia; : PC - parcialmente cheia; : PV - parcialmente vazia; : V - vazia; NI - não identificado.

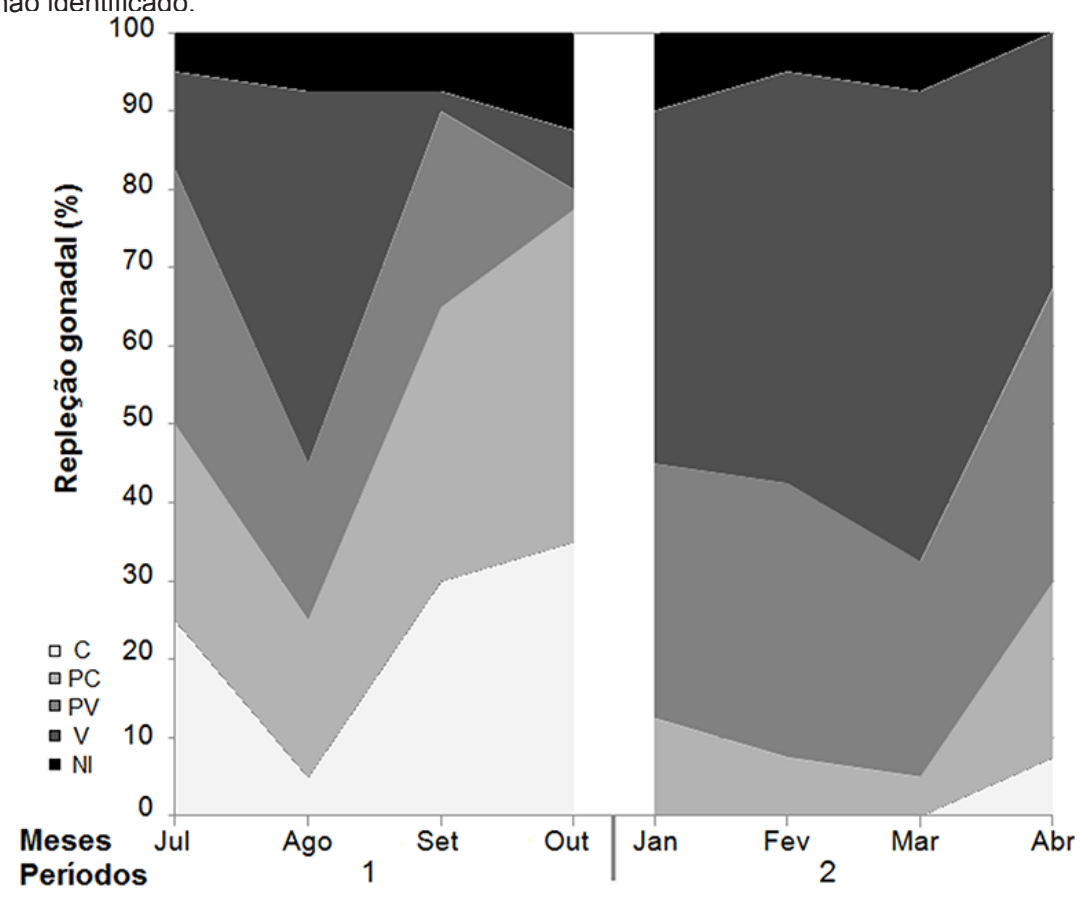


1758), foi identificada também na região nordeste do Brasil uma menor presença de posturas no período seco (julho a dezembro) e maior presença de posturas no período chuvoso do ano (janeiro a maio de 1991) (Matheus-Cascon \& Martins, 1999).

Santos \& Boehs (2011), efetuando estudos de estrutura de população do neogastrópode $T$. haemastoma em llhéus (Bahia), observaram recrutas ao longo de todo o ano, evidenciando uma reprodução contínua. Rocha-Barreira (2010) verificou, na Praia do Cassino (RS), sul do Brasil, no gastrópode Olivancillaria vesica auricularia (Lamarck, 1810), maturação gonádica de setembro a dezembro seguido por desovas de janeiro a abril. Essa mesma autora constatou repouso reprodutivo durante o Outono e o Inverno para a espécie estudada. Nos resultados obtidos na llha do Mel, pôde-se identificar a presença de exemplares de $L$. flava com gônadas repletas (estágios cheio e parcialmente cheio) em todos os meses, o que indica uma característica de reprodução contínua da espécie no litoral do Paraná.

Dentre os fatores que influenciam a sustentabilidade de espécies de gastrópodes, pode-se destacar alterações das características reprodutivas e fisiológicas dos organismos. Uma destas alterações é atribuída a contaminação de regiões portuárias por compostos de tributil-estanho (TBT), que têm sido utilizados como biocidas na redução da bioincrustação (fouling) de embarcações. O TBT pode promover o desenvolvimento de um pênis não funcional nas fêmeas, evento a que se denomina de imposex (Garaventa et al., 2006). No Brasil, o monitoramento deste poluente é efetuado predominantemente com o neogastropoda T. haemastoma (Biasi et al., 2010; Castro et al., 2000; 2007a; 2007b; 20012). Em estudos reprodutivos, alterações na proporção sexual (decorrentes de castração das fêmeas) podem indicar influência deste contaminante na reprodução da espécie alvo (Broeck et al., 2009). As alterações na proporção sexual identificadas no costão rochoso da Ilha do Mel, podem estar associadas a contaminantes oriundos do Centro de Resíduos da llha do Mel, do fluxo de embarcações para o trapiche da Vila das Encantas e do transporte de resíduos para a cidade de Paranaguá (efetuados por uma embarcação que fica aportada a frente do costão estudado). Broeck et al. (2009), ao estudarem L. littorea, identificaram imposex e posteriormente a castração de fêmeas decorrentes do bloqueio dos gônodutos, situação similar a castração decorrente por parasitismo.

Estudos realizados por Castro et al. (2007) na Ilha do Mel, atestaram que $85 \%$ das fêmeas de T. haemastoma estavam imposexadas. Esses autores constataram fluxo naval de 2.252 navios no ano de 2006 . Nesta mesma região, em estudo posterior (Castro et al., 2012) constataram valores de TBT de 52,6ng/g de tecido em $T$. haemastoma. Embora essas análises ainda não tenham sido feitas para $L$. flava, as proporções sexuais observadas nos resultados do presente estudo sugerem que o mesmo deve estar ocorrendo para esta espécie. Para comprovar esta possível influência dos contaminantes provenientes das embarcações do CEP sobre a reprodução de $L$. flava na região, se fazem necessários estudos de frequência, intensidade de imposex e análise histoquímicas das gônadas para caracterizar a possível castração das fêmeas nessa espécie.

\section{REFERÊNCIAS}

Bauer, B.; Fioronil, P.; Ide, I.; Liebe, S.; Oehlmann, J.; Stroben, E. \& Watermann, B. 1995. TBT effects on the female genital system of Littorina littorea: a possible indicator of tributyltin pollution. Hydrobiologia 309 (1-3):15-27.

Biasi, J.B.; Tomás, A.R.B. \& Imparato, L. 2010. Imposex in the whelk Stramonita haemastoma (Neogastropoda: Muricidae), from Baixada Santista (SP), Brazil. Bioikos, Campinas 24 (1): 5-12.

Borkowski, T. M. 1971. Reproduction and reproductive periodicities of south floridian littorinidae (Gastropoda: Prosobranchia). Bull. Mar. Sci. 21 (4): 826-840.

Broeck, H. V.; Wolf, H.; Backeljau, T. \& Blust, R. 2009. Comparative assessment of reproductive impairment in the gastropod mollusk Littorina littorea along the Belgian North Sea coast. Sci. Total Environ. 407: 3063-3069.

Castro, B.I.; Matthews-Cascon, H. \& Fernandez, M. A. 2000. Imposex em Thais haemastoma (Linnaeus, 1767) (Mollusca: Gastropoda) uma Indicação da Contaminação por Organoestânicos na Costa do Município de Fortaleza - Ceará - Brasil. Arq. Ciên. Mar. 33: 143-148.

Castro, I. B.; Bemvenuti, C. E. \& Fillmann, G. 2007a. Preliminary Appraisal of Imposex in Areas Under the Influence of Southern Brazilian Harbors. J. Braz. Soc. Ecotoxicol. 2 (1):73-79.

Castro, I. B.; Alves De Lima, A. F.; Braga, A. R. C. \& Rocha-Barreira, C. A. 2007b. Imposex in Two Muricid Species (Mollusca: Gastropoda) from the Northeastern Brazilian Coast. J. Braz. Soc. Ecotoxicol. 2 (1): 81-91.

Castro, I. B.; Rossato, M. \& Fillmann, G. 2012. Imposex reduction and residual butyltin contamination in Southern Brazilian harbors. Environ. Toxicol. Chem. 31 (5): 947-954. 
Garaventa, F.; Faimali, M. \& Terlizzi, A. 2006. Imposex in pre-pollution times. Is TBT to blame? Mar.Poll. Bull. 52: 696-718.

Hyman, L. H. 1967. Capítulo VII: Class Gastropoda: Introduction. Hyman, L. H. (ed) In: The Invertebrates: Mollusca I. Volume VI. Aplacophora, Polyplacophora, Monoplacophora, Gastropoda - The coelomate Bilateria. MacGraw-Hill Book Company. 152-169pp.

Maack, R. 1981. Geografia física do Estado do Paraná. Curitiba, J. Olympio/Secretaria da Cultura e Esporte do Estado do Paraná. 450pp.

Matthews-Cascon H. \& Martins, I. X. 1999. Notas sobre a reprodução de Neritina virgínea (LINNAEUS, 1758) no Nordeste do Brasil (Mollusca: Gastropoda: Neritidae). Arq. Ciên. Mar 32: 129-132.

Noernberg, M. A.; Angelotti, R.; Caldeira, G.A. \& Ribeiro de Sousa, A.F. 2008. Environmental sensitivity assessment of Paraná coast for oil spill. Braz. J. Aquat. Sci. Technol. 12 (2): 49-59.

Nybakken, J. W. Marine Biology. 1997. An ecological approach. Addison-Wesley Educational Publishers, Inc. $477 p p$.

Rios, E. C. 2009. Compendium of Brazilian Sea Shell - FURG, Rio Grande, RS. 676pp. Rocha-Barreira, C. A. 2002. Gonad Characterization and Reproductive Cycle of Collisella subrugosa (ORBIGNY, 1846) (Gastropoda: Acmaeidae) in the Northeastern Brazil. Braz. J. Biol. 62 (4B): 885-895.
Rocha-Barreira, C. A. 2010. Ciclo reprodutivo de Olivancillaria vesica auricularia (Lamarck, 1810) (Mollusca: Gastropoda: Olividae) no Sul do Brasil. Arq. Ciên. Mar 43 (2): 110 - 118.

Santos, J. J. B. \& Boehs, G. 2011. Spatial-temporal distribution and recruitment of Stramonita haemastoma (Linnaeus, 1758) (Mollusca) on a sandstone bank in llhéus, Bahia, Brazil. Braz. J. Biol. 71 (4): 799-805.

Simone, L. R. 1998. Morphological study on Littorina flava (KING \& BRODERIP) from Brazil (Caenogastropoda, Littorinidae). Revta Bras. Zool. 15 (4): $875-887$.

Schena, F. 2006. Turismo, estado, sociabilidades e mudança: uma etnografia da vila de Encantadas, Ilha do Mel. Dissertação (Mestrado) - Setor de Ciências Humanas, Letras e Artes, Universidade Federal do Paraná - Curitiba. 107p.

Scrosati R. \& Heaven C. 2007. Spatial trends in community richness, diversity, and evenness across rocky intertidal environmental stress gradients in eastern Canada. Mar. Ecol. Prog. Series 342: 1-14.
Submetido: Agosto/2013 Revisado: Fevereiro/2014 Aceito: Março/2014 